\title{
Ethnologies
}

\section{Habiter la terre}

\section{Une lecture de l'Acte d'indépendance d'Haiiti}

\section{Jean François}

Volume 28, numéro 1, 2006

Haïti - Face au passé

Haïti - Confronting the Past

URI : https://id.erudit.org/iderudit/014151ar

DOI : https://doi.org/10.7202/014151ar

Aller au sommaire du numéro

\section{Éditeur(s)}

Association Canadienne d'Ethnologie et de Folklore

ISSN

1481-5974 (imprimé)

1708-0401 (numérique)

Découvrir la revue

Citer cet article

François, J. (2006). Habiter la terre : une lecture de l'Acte d'indépendance d'Haïti. Ethnologies, 28(1), 119-132. https://doi.org/10.7202/014151ar
Résumé de l'article

L’Acte d'indépendance d'Haïti n'est jamais questionné ; archivé une fois pour toutes, il souscrit à la norme du registre : acte de naissance, de baptême, de mariage... Pourtant, son rédacteur, Boisrond-Tonnerre, n'avait pas à sa disposition un formulaire à remplir, à la manière de l'officier d'état civil. Il se devait de produire son discours. Ce qui en est ressorti, c'est une vision du monde dont lui et ses contemporains n'étaient pas forcément conscients. Le simulacre dépasse la proclamation de naissance de l’État haïtien, il légitime l'entrée sur la terre d'une nouvelle catégorie d'hommes, dont l'argument essentiel devient le sang, un sang attribué à tort à Boisrond-Tonnerre pour sa célèbre déclaration du 31 décembre 1803, mais qui n'est autre que la caution à l'intégration de l'état humain. De toutes les instances de cet univers sémiotique, deux seulement peuvent s'octroyer sans ambages le poste de sujet l'officiant, rôle que Boisrond s'est attribué, et le général en chef, véritable sujet-destinateur qui commande aux généraux de renoncer à la France pour le bonheur du pays. L'Acte d'indépendance d'Haïti est ici mis en comparaison sur ce plan sémiotique avec la Déclaration d’indépendance des États-Unis et avec la déclaration des Droits de l’homme en France.
Ce document est protégé par la loi sur le droit d'auteur. L'utilisation des services d’Érudit (y compris la reproduction) est assujettie à sa politique d'utilisation que vous pouvez consulter en ligne.

https://apropos.erudit.org/fr/usagers/politique-dutilisation/ 


\title{
HABITER LA TERRE
}

Une lecture de l'Acte d'indépendance d'Haïti

\author{
Jean François \\ York College, City University of New York
}

J'ai commencé à m'intéresser à l'Acte d'Indépendance quand je me suis rendu compte que je ne l'avais pas lu. Ma situation, il me semble, n'est pas singulière, puisque dans le parcours de l'écolier haïtien, l'Acte ${ }^{1}$ est supplanté par un autre acte de parole, oral celui-ci : la célèbre déclaration de Boisrond-Tonnerre. Il faut croire que, même à l'école, on se fait un devoir de nous rappeler que nous vivons dans une société d'oralité et qu'en conséquence, l'écrit est pour les archives. J'exagère un peu. Une autre interprétation est peut-être plus pertinente : l'Acte, document juridico-administratif, a cédé le pas à un texte littéraire. $\mathrm{Si}$ cette proposition a une certaine validité, il conviendra de reconnaitre en Boisrond-Tonnerre le premier écrivain haïtien, non le premier écrivant qu'il est à coup sûr, mais le premier artisan de la langue, dont l'œuvre se réduit à un énoncé allégorique: "Pour dresser l'acte de notre indépendance, il nous faut la peau d'un blanc pour parchemin, son crâne pour écritoire, son sang pour encre et une baïonnette pour plume » (Ardouin 1958, Tome VI : 7). La phrase si bien tournée a séduit Dessalines, qui a désigné Boisrond-Tonnerre pour être le rédacteur de l'Acte. Pourtant, on sait que la décision du général en chef de l'armée indigène ne peut suffire à définir la fonction de l'Acte. Le texte est appelé à exécuter deux programmes corrélés, dont le second subsume le premier : l'institution de l'État haïtien et une prise de possession de la terre. La création de l'État haïtien signe, en effet, l'entrée d'un nouveau

1. Pour simplifier, l'Acte avec A majuscule renvoie dans cette étude à l'acte d'indépendance. 
groupe d'hommes dans le monde ${ }^{2}$ : le Code noir ne rangeait-il pas les Noirs dans la catégorie du bétail ? On comprend que, dans ce cas, le $1^{\text {er }}$ janvier consacre symboliquement pour les Haïtiens leur droit d'habiter la terre.

C'est la mise en place de cette double performance que je me propose d'interroger en prenant appui sur la grammaire modale de Jean-Claude Coquet : Le Discours et son sujet $(1984,1985)$ et La quête du sens (1997).

La grammaire modale est une approche sémiotique qui cherche à cerner l'univers de signification à partir de l'histoire transformationnelle des actants. Loin de se limiter au plan descriptif des actions caractérisé par le faire, l'être, l'avoir, elle établit un plan des modalités. Celles-ci sont considérées comme les grandes catégories logiques de la subjectivité. Elles régissent les actions dans une structure narrative, c'està-dire que d'elles dépendent les verbes descriptifs. Elles sont au nombre de quatre : le savoir, le pouvoir, le vouloir et le devoir.

Ces principaux «domaines distincts», je les dénommerai, pour reprendre une expression de J. B. Grize, « cognitif» (le savoir), « pragmatique », (le pouvoir), «volitif » (le vouloir) et « déontique » (le devoir) (Coquet 1997 : 149).

De là prend naissance une morphologie actantielle fondée sur une combinatoire des trois premières modalités; le vouloir en est l'axe déterminant. Sa présence ou son absence fait de l'actant un sujet ou un non-sujet. L'actant sujet est engagé dans un programme dont il évalue les différentes étapes, alors que l'actant non sujet, privé de jugement, n'exécute que ce pour quoi il était programmé. À côté de sa morphologie actantielle, le sujet est appelé à s'identifier pour chaque programme à partir d'une dimension modale. Ainsi, il peut être sujet épistémique, pragmatique, etc. Les programmes tiennent leur unité de leur isotopie, c'est-à-dire du plan de signification qui les sous-tend. "On voit qu'une définition fondée sur les modalités nous conduit à combiner traits génériques (dimension et isotopie) et traits spécifiques (suite ternaire)» (1984: 29).

La modalité du devoir vient toujours en surdétermination : elle place l'actant dans une relation hétéronome par rapport à un destinateur; le sujet autonome n'y est pas astreint.

2. Cette entrée est glosée de différentes manières : première république noire, premier peuple noir, etc. 
L'économie de cette sémiotique est qu'elle permet d'élucider les différentes étapes du parcours d'un acteur, eu égard au rôle actantiel que chaque programme lui commande. Aussi s'avère-t-elle une grille efficace pour l'appréhension de textes apparemment simples — pourtant retors —, comme l'Acte d'indépendance d'Haïti.

Acte d'indépendance

Aujourd'hui, premier janvier mil huit cent quatre, le Général en chef de l'armée indigène, accompagné des généraux, chefs de l'armée, convoqués à l'effet de prendre les mesures qui doivent tendre au bonheur du pays;

Après avoir fait connaître aux généraux assemblés ses véritables intentions d'assurer à jamais aux indigènes d'Haïti un gouvernement stable, objet de sa plus vive sollicitude, ce qu'il a fait par un discours qui tend à faire connaître aux puissances étrangères la résolution de rendre le pays indépendant et de jouir d'une liberté consacrée par le sang du peuple de cette île ; après avoir recueilli leur avis, a demandé que chacun des généraux assemblés prononçât à jamais le serment de renoncer à la France, de mourir plutôt que de viure sous sa domination, et de combattre jusqu'au dernier soupir pour l'indépendance.

Les généraux, pénétrés de ces principes sacrés, après avoir donné d'une voix unanime leur adhésion au projet bien manifeste d'indépendance, ont tous juré à la postérité, à l'univers entier, de renoncer à jamais à la France, et de mourir plutôt que de viure sous sa domination.

Fait aux Gonaïves, ce $1^{\text {er }}$ janvier 1804 et le $1^{\text {er }}$ de l'Indépendance d'Haïti.

Signé : Dessalines, général en chef; Christophe, Pétion, Clervaux, Geffrard, Vernet, Gabart, généraux de division; P. Romain, E. Gérin, F. Capoix, Daut, J. L. François, Férou, Cangé, L. Bazelais, Magloire Ambroise, J. J. Herne, Toussaint Brave, Yayou, généraux de brigade; Bonnet, F. Papalier, Morely, Chevalier, Marion, addjudants-généraux; Magny, Roux, chefs de brigade; Charéron, B. Loret, Qenez, Makajoux, Dupui, Carbonne, Diaquoi aîné, Raphaël, Mallet, Derenoncourt, officiers de l'armée ; et Boisrond Tonnerre, secrétaire. 


\section{Destinateur et sujet : l'hétéronomie}

Derrière sa disposition en trois paragraphes, notre texte ne comporte que deux phrases grammaticales. Ce morcellement n'est pas surprenant ; c'est à peu près le schéma de tous les actes officiels : acte de naissance, acte de mariage... La première phrase énonce un sujet grammatical, le général en chef de l'armée indigène, qui attend la fin de la phrase pour se conjoindre avec un verbe : "a demandé ». L'écart est comblé par un ensemble de propositions dépendantes greffées sur la seule principale, elle-même allongée par des compléments infinitifs :

Aujourd'hui, premier janvier mil huit cent quatre, le Général en chef de l'armée indigène... a demandé que chacun des généraux assemblés prononçât à jamais le serment de renoncer à la France, de mourir plutôt que de vivre sous sa domination, de combattre jusqu'au dernier soupir pour l'indépendance.

Une injonction est faite qui ouvre une alternative : les généraux doivent obéir ou rejeter le commandement du général en chef. Ils obéissent. C'est le propos de la deuxième phrase : "Les généraux... ont tous juré à la postérité, à l'univers entier, de renoncer à jamais à la France, et de mourir plutôt que de vivre sous sa domination ».

Ces deux mouvements paraissent à première vue résumer le trajet discursif de l'Acte. En nous référant aux propositions indiquées plus haut, nous pouvons avancer quelques considérations.

D'abord, ce programme se place sur l'isotopie du pouvoir et est en accord avec la dimension du général en chef. Celui-ci est en position décalée par rapport à l'actant collectif, "les généraux ». La particule « en chef » accolée à son titre établit une logique des places qui consacre sa supériorité. La modalité du pouvoir qui le caractérise est contrebalancée par celle du devoir des généraux; ils doivent être considérés comme des sujets hétéronomes. En d'autres termes, le général en chef a un statut de destinateur par rapport à l'actant collectif « les généraux ». Il le libère d'un autre destinateur qui est la France ; il est plutôt un anti-destinateur.

Mais l'action des généraux en soi mérite attention : renoncer à la France réside dans le fait de le dire. C'est un acte de parole performatif ${ }^{3}$.

3. «Les énoncés performatifs... ont la propriété de pouvoir dans certaines conditions accomplir l'acte qu'ils dénomment, c'est-à-dire de faire quelque chose du seul fait de le dire : énoncer je te promets de venir, c'est ipso facto accomplir un acte, celui de promettre »(Charaudeau et Mainguenau 1997: $16-17)$. 
Et la performance passe par l'introduction de deux nouveaux actants : la postérité et l'univers. Ces actants définissent un ordre des choses transcendant; Coquet les appelle tiers actants ${ }^{4}$. Leur introduction est nécessaire à la dépossession de la France. Le renoncement n'est effectif que s'il est communiqué à la postérité et à l'univers. Or la performance est au profit d'un destinataire énoncé clairement : le pays. Une première conclusion peut être anticipée : Haïti ne relaie pas encore la France ; la délégation de pouvoir se fera plus tard par le général en chef.

\section{Une autonomie douteuse}

J'ai simplifié à dessein jusque-là pour pouvoir dégager le programme dominant de l'Acte. Mais on ne peut pas réduire son réseau discursif à la simple isotopie pragmatique. Sous-jacent se développe un autre programme situé sur l'isotopie du savoir et qui semble placer les acteurs dans l'autonomie. En réintégrant les propositions subordonnées dans le texte, on le découvrira.

La réinsertion des temporelles introduites par «après avoir»va offrir un nouvel éclairage. Le général en chef paraît, dès le début, entrer dans une relation d'échange avec les généraux. L'objet de valeur échangé est un objet cognitif : le général expose son intention aux généraux et les invite à l'évaluer. Le plan épistémique est marqué par le syntagme verbal «avoir fait connaître » auquel fait pendant « après avoir recueilli leur avis ». Le général en chef donne aux généraux et reçoit d'eux : il y a don et contre-don. C'est un programme de contrat, qui est bouclé avec la troisième temporelle : " après avoir donné leur adhésion au projet d'indépendance ». L'adhésion relève avant tout du vouloir, modalité fondatrice de l'actant sujet. Le fait par le général en chef de la recevoir implique la reconnaissance du statut de sujet des généraux. Dans ce programme semblent donc présentés deux sujets de droit ${ }^{5}$. Et

4. «Le postulat de la sémiotique subjectale est que l'organisation transphrastique ne repose que sur trois instances d'énonciation possibles : le sujet, le non-sujet et le tiers actant» (Coquet 1997 : 154).

5. Le sujet de droit est fort d'une identité reconnue, fruit d'un parcours effectué, contrairement au sujet de quête qui s'inscrit dans un processus d'affirmation. Le premier est un sujet du contrat et le deuxième un sujet du désir. La morphologie actantielle des deux actants ici prend la forme d'une assomption ; il y a une visée constative où l'identité n'est plus à démontrer qui peut s'écrire sous la forme « Spv »; chaque lettre représente une modalité, la majuscule la dimension de l'actant. Le vouloir placé à la fin de la suite ternaire marque l'assomption (voir Coquet 1983 : chap. II). 
ce droit n'est fonction d'aucune hypothèque, il est entier, il repose sur l'armée indigène, qui a combattu et vaincu l'armée française. Cette armée préexiste à la date du $1^{\mathrm{er}}$ janvier 1804 . On peut déjà tenter une comparaison avec la Déclaration d'indépendance des États-Unis, où les protagonistes sont les représentants du peuple, alors que le peuple ne sera créé que par la performance du discours. Si Derrida peut parler d'acte différantiel (1984: 24) à propos de ce dernier document, il n'en est pas question, à ce niveau d'analyse, pour l'Acte : l'État haïtien est créé par des militaires d'une armée instituée.

Gardons en tout cas à l'esprit que, pour le rédacteur de l'Acte, la constitution d'un État doit passer par un projet épistémique. Mais un tel projet n'est-il pas trompeur? En avons-nous jusque-là démêlé toutes les intrications?

Le texte nous révèle ce que donne le général en chef, dans ses moindres détails. La tautologie est de mise : ses intentions consistent à « assurer à jamais aux indigènes d'Haïti un gouvernement stable » (la constitution d'un État). L'explicative introduite par les deux points décrit la performance : " ce qu'il a fait par un discours qui tend à faire connaître aux puissances étrangères la résolution de rendre le pays indépendant et de jouir d'une liberté consacrée par le sang du peuple de cette île ».

Si on met de côté l'aspect approximatif de l'expression ${ }^{6}$, on trouvera dans le discours du général le même trait performatif que le renoncement des généraux. Mais le scénario est différent : ceux-ci s'adressaient à l'univers et à la postérité, alors que le général en chef s'adresse aux généraux et atteint en même temps les puissances étrangères. L'évocation du tiers actant univers n'est pas exclue non plus du discours (qu'on pense au terme sang, dont je parlerai plus loin).

Mais le contre-don des généraux n'a pas droit à cette élaboration ; il reste un terme abstrait : l'avis. En quoi consiste-t-il ? L'avis en question ne peut être que l'approbation de la position du général, l'adhésion au projet d'indépendance - cela est d'autant plus évident que le terme " résolution » associé à l'article défini avait déjà signifié que la position du général en chef n'était pas à remettre en question. Du coup, nous

6. La clausule est ratée, quand on considère le cumul des deux infinitifs en position de complément à « résolution » : quelle est cette instance qui veut jouir de la liberté consacrée par le sang du peuple? 
sommes autorisés à questionner la transformation opérée par le général en chef sur les généraux. N'est-ce pas une transformation du même au même ? Les généraux sont réunis pour faire ce qu'ils ont à faire : donner leur avis favorable. Si cette hypothèse est acceptable, leur statut de sujet est ruiné. Car le sujet est par définition un actant auquel est associé un nombre non limité de prédicats ${ }^{7}$; il peut les multiplier jusqu'à arriver à son but, contrairement au non-sujet qui est programmé.

Dans de telles conditions, l'Acte met en scène un actant individuel dont le savoir accouplé au pouvoir lui permet d'entraîner après lui un actant collectif. Le général en chef est, en effet, assez puissant pour subsumer tout le savoir et tout le pouvoir de son vis-à-vis, les généraux. Mais une telle orientation détourne-t-elle le texte de sa mission première qui est celle de légitimer le peuple haïtien?

\section{L'Autre possible}

Habiter est un être-là, il ne peut se faire dans la solitude. Je suis là à l'idée que d'autres sont aussi là pour réfracter ma présence. Je deviens homme quand d'autres hommes peuvent en attester. Même en voulant changer ma manière d'habiter, je me dois de convoquer l'Autre. Le simulacre $^{8}$ identitaire ne peut relever que de la reconnaissance de la part de l'Autre. S'affirmer nécessite donc l'instauration d'un réseau d'interlocution dont l'Autre ${ }^{9}$ constitue un pôle. Il faut qu'il vienne assumer ma présence à moi, il lui faut en prendre acte en tant que personne physique. C'est la problématique du Soi-même comme un autre débattue par Paul Ricœur (1990). Ainsi, l'Acte doit négocier l'entrée des Haïtiens sur la terre à partir d'un rituel fondateur ; cette tâche est largement assumée par l'instance énonçante d'origine ${ }^{10}$, le sujet épistémique qui construit le système des valeurs.

7. Le sujet peut multiplier les prédicats dans la mesure où il est maître de ses actions ; il évalue constamment sa situation, alors que le non-sujet correspond à un nombre limité de prédications : il n'a aucune emprise sur son sort.

8. Ce mot n'a rien de péjoratif dans notre vocabulaire ; il implique tout simplement l'idée de rite.

9. Notons que l'instance de l'Autre a été déjà convoquée à travers le vocable indigène : se reconnaître c'est se placer dans la relation.

10. "J'appelle discours une organisation transphrastique (cette visée est inconnue des linguistes stricto sensu) rapportée à une ou plusieurs instances d'énonciations » (Coquet 1997 : 148). C'est fort de cette définition du discours que Coquet identifie dans tout discours une instance énonçante d'origine. 
Elle s'affirme dès l'incipit à travers son champ positionnel, c'est-àdire ses coordonnées de temps, d'espace et de personne. Le terme " aujourd'hui », premier mot de l'acte, est un marqueur ${ }^{11}$ de présence très fort. Associé à la date «premier janvier 1804 », il insère ce moment unique dans le temps calendaire. Les instances narratives évoquées par la suite ne servent qu'à renforcer la présence du « je » de l'énonciation, puisqu'elles sont introduites par des articles définis à valeur démonstrative : "le général », "les généraux », "l'armée indigène ». Ce même article défini dans sa forme contractée délimite le centre spatial : "du pays ». Dès lors, le champ est suffisamment posé pour marquer cette entrée unique dans le monde. Les repères sont déjà en eux-mêmes performatifs puisqu'ils créent le pays. Il suffit, en effet, de dire le pays pour que celui-ci accède à l'existence. L'accession s'est faite en un temps, en un lieu et avec des personnes précises. Ce qui reste à faire accepter, c'est l'établissement de ce pays nouveau sur la terre.

Le premier outil de la construction est le discours indirect ; ce relais de parole permet de produire une interprétation, ou plutôt un traitement des actes de parole, à partir d'une évaluation implicite. De là s'explique que l'avis des généraux n'est jamais présenté alors que celui du général en chef est largement commenté. Encore faut-il être sûr que le discours indirect ne bascule pas dans le discours indirect libre: on ne sait pas si le terme « objet de sa plus vive sollicitude » appartient au général en chef ou au narrateur, mais on retient qu'il consacre la volonté inébranlable de celui-là.

La liberté interprétative que s'octroie le narrateur conduit à la convocation d'un premier actant nécessaire à la proclamation d'indépendance : les puissances étrangères. Cet actant, rappelons-le, n'est pas sur le même plan que la France. C'est un actant collectif dont celle-ci est un membre. La fondation de l'État haïtien doit s'inscrire dans une garantie de reconnaissance de la part des puissances qui dirigent le monde. L'insertion de cet actant institue un circuit de la conversation éclatée, dans la mesure où il repose sur la ternarité, non sur la binarité : d'un côté, il y a les généraux, allocutaires directs du message, et de l'autre les puissances étrangères qui sont visées par ricochet, dirait-on.

Mais la convocation est bien singulière : comment accuser la présence des puissances étrangères dans l'espace gonaïvien en ce jour

11. Ces marques formelles de l'énonciation, connues traditionnellement sous le nom de déictiques, Roman Jakobson les appelle des embrayeurs ( 1963 :176). 
du $1^{\text {er }}$ janvier 1804 alors qu'elles sont de fait absentes ? Le handicap est contourné facilement par l'utilisation du lexème modalisateur «tendre ». Tendre est une évaluation hypothétique; il nécessite comme premier critère la durée. Il offre ainsi l'économie de maintenir la cohérence argumentative.

En fait l'Autre, c'est nous dans l'Acte. Nous engagés dans un processus d'accréditation. Nous sommes l'Autre possible, celui pour lequel il n'existe pas encore de repère et que la terre doit accueillir. L'argumentation ne peut donc se construire que sur un univers uniplanaire (Coquet 1984 : chap. III) : renoncer à la France ou ne pas exister. Renoncer à la France, c'est libérer le lieu, faire en sorte qu'il ne soit à personne pour que nous puissions l'habiter. Et notre légitimité est assurée par la garantie d'un ordre des choses, par l'autorisation du tiers actant universel, une forme de lettre de créance.

Voilà le message adressé aux puissances étrangères. L'indépendance, notre acceptation sur la terre, ne se négocie pas à partir d'une référence personnelle, la parole d'un « je », ni sur la position d'une doxa, le point de vue de cette communauté qui réclame l'indépendance, la parole d'un " on ", mais bien sur un ordre transcendant, celui que Coquet appelle le «il »(1984; chap. III), point d'orgue de la véridiction. Il est donc normal que les généraux à qui est réservée la dernière performance jurent à l'univers leur renoncement à la France.

Une telle compétence argumentative était nécessaire pour la rédaction du document. L'Acte doit négocier avec les puissances qui dirigent le monde. L'exemple de cette négociation a déjà été fourni par les deux parents proches: la déclaration des Droits de l'homme et du citoyen ( "tous les hommes naissent et demeurent égaux en droit» stipule l'article un) et la Déclaration d'indépendance des États-Unis d'Amérique ( $Q$ Quand dans le cours des événements humains... » dit l'incipit). Mais ici, ce n'est pas le lieu de rappeler ce qui est redevable à tous les hommes; l'urgence est dans l'affirmation de son humanité. Le moment des Haïtiens est décalé par rapport à celui des Américains et des révolutionnaires français. Cette particularité de l'Acte semble même inscrite dans l'histoire de sa rédaction.

On sait que Charéron, qui en avait rédigé la première version, s'était largement inspiré des Déclarations de 1776 et de 1789. Le travail lui avait pris près d'un mois, mais il avait été rejeté par Dessalines. BoisrondTonnerre, lui, a écrit la sienne dans la nuit du 31 décembre 1803 au $1^{\text {er }}$ 
janvier 1804. Celle-ci n'a fait l'objet d'aucune épreuve, puisque le rédacteur, épuisé par son travail, a sombré dans un sommeil assommant au point qu'il est arrivé en retard sur la place d'armes des Gonaïves ${ }^{12}$.

Comment peut-on s'expliquer le rejet de Charéron par Dessalines ? Selon Madiou, le document manquait de chaleur. La fameuse déclaration spontanée de Boisrond-Tonnerre a retrouvé la note d'exaltation qui caractérisait les pères fondateurs de la nation ; Ardouin $^{13}$ ne contredit pas ce point de vue. Tout en blâmant Boisrond pour ses propos «sanguinaires ", il reconnaît qu'ils se justifiaient par l'air du temps, ce à quoi Charéron n'avait pas pu souscrire.

Si l'on doit faire confiance à ces jugements, il faudra soulever la question du sang. La performance du rédacteur devait donc intégrer le sang. On s'étonne que nos historiens se soient attardés sur ce sang de la fameuse déclaration sans se questionner sur le sang de l'Acte. À qui appartient-il ? Boisrond-Tonnerre, lui, savait que sa boutade ne pouvait être que l'envers de l'Acte. Il est écrit avec le sang du peuple: je ne peux pas habiter la terre avec le sang de l'Autre. Même si celui-ci n'est pas loin, car le peuple qui s'institue le $1^{\text {er }}$ janvier 1804 n'est en mesure de revendiquer son sang que parce qu'il a pu verser le sang français. Mon sang doit être la caution nécessaire à mon acceptation par l'Autre. Ce n'est qu'à ce moment que je peux être moi, que je peux accéder à un circuit d'interchangeabilité avec lui, en bref, être lui aussi, sinon la performance est ratée. Alors, la rage qu'on lui réclamait, il l'a de préférence versée dans la proclamation de Dessalines, qu'il a aussi rédigée et lue le $1^{\text {er }}$ janvier 1804 . C'est vraisemblablement ce discours que l'acte relaie ${ }^{14}$.

12. Madiou (1983 : 145), dont l'écriture de l'histoire prend souvent la forme d'un récit mythologique, va jusqu'à affirmer qu'il avait fallu défoncer la porte de sa maison pour pouvoir réveiller Boisrond; c'est le sommeil du héros qui vient de tuer le dragon.

13. «Le 31 décembre, Charéron lut son travail pour Dessalines, en présence des généraux et de tous les autres officiers. La cérémonie de la déclaration d'indépendance devait avoir lieu le lendemain, $1^{\text {er }}$ janvier 1804, pour commencer l'ère nouvelle avec la nouvelle année. Ce long exposé de faits et de principes produisait une impression fâcheuse sur l'esprit de Dessalines, qui était ardent et animé de vengeance. Il manifesta une désapprobation formelle de l'œuvre» (Ardouin 1958, Tome III : 9).

14. On sait que Dessalines a aussi improvisé un discours en créole avant la lecture de sa proclamation par Boisrond-Tonnerre. 
Le terme «sanguinaire» de Beaubrun Ardouin, émis avec toute sa charge de négativité, est loin de la problématique de notre texte, qui valorise plutôt le martyre. La tâche ne consiste pas ici à renverser une hiérarchie humaine imposée, un ordre social, en vue d'instituer la République; on institue plutôt son humanité. Quoi de plus indiqué pour l'affirmer que le métonyme par excellence de l'état humain : le sang. C'est à la vérité le principal support argumentatif de l'Acte ; ceci l'éloigne de la déclaration des Droits de l'homme qui énumère toute une série de droits inhérents à l'être humain, comme il l'éloigne de la Déclaration d'indépendance des États-Unis où sont consignés un ensemble de délits commis par le roi pour justifier la rupture avec la Grande Bretagne.

\section{Boisrond-Tonnerre, rédacteur et officiant}

Mais tout cela ne doit pas nous faire perdre de vue que BoisrondTonnerre a su tirer parti de sa position de rédacteur solitaire.

À strictement parler, la narration de l'Acte se place dans l'antériorité, puisqu'il a été rédigé avant les événements qu'il raconte. Il suffisait, par exemple, que le jour du $1^{\text {er }}$ janvier 1804 un incident perturbant, comme le retour de l'armée française, vint empêcher la tenue de la cérémonie pour que le document fût annulé ou soumis à modification. Pourtant, il n'en est rien ; c'est le sort de tout texte performatif : l'Acte est condamné à s'énoncer en se lisant, il s'institue en se faisant et institue du même coup le peuple haïtien. C'est là sa valeur différantielle, pour rester dans la terminologie de Derrida. Il y a soumission de l'ordre du discours à celui des événements. Car nous avons devant nous un de ces textes dont l'écriture est antérieure à l'énonciation. Le prêtre qui dit : «je vous déclare mari et femme " ne fait que réciter une phrase apprise. Mais elle n'aura d'effet que si les circonstances sont réunies pour son exploitation, à savoir un homme et une femme devant une assistance avec tout ce que cela comporte de rites pour pouvoir arriver au mariage.

Ainsi, le discours de l'Acte ne peut avoir de date que le $1^{\text {er }}$ janvier 1804, au moment où Boisrond-Tonnerre le lit sur la place d'armes des Gonaïves. Cela nous autorise à dire que l'instance énonçante d'origine de l'Acte c'est bien lui, Boisrond-Tonnerre. Il lui était confié dans la journée du 31 décembre 1804 un double rôle : celui de rédacteur et de lecteur. Mais comme les conditions de la rédaction n'étaient pas définies, il en a profité pour faire du lecteur l'instance d'énonciation. Je 
m'explique : à partir du moment où il a décidé en solitaire de ne pas attribuer l'énonciation à un actant collectif, le « Nous, les représentants du peuple» de la Déclaration d'indépendance des États-Unis, qui se formulerait sous la forme "Nous, les généraux de l'armée indigène » et qui placerait Dessalines sur un plan d'égalité avec les autres généraux, il a érigé un "je » d'énonciation qui ne peut être que le lecteur du texte performatif — le « Je soussigné Dessalines... » ferait accroc. Son rôle est celui du prêtre qui proclame le mariage. Mais il ne faut pas y voir une forme d'usurpation, une sorte de vedettariat volé aux autres. Le programme qui lui a été confié par Dessalines ne prête à aucune équivoque: "Boisrond, je te charge d'exprimer au peuple mes sentiments à l'égard des Français» (Madiou 1983: 145).

Et Boisrond-Tonnerre, lecteur et énonciateur de l'Acte, s'est vu dans la position du prêtre pour proclamer l'indépendance. Mais l'Acte déjà signé par les officiers haut gradés de l'armée indigène ne crée pas encore l'État. Il faut une dernière signature : celle du peuple massé sur la place d'armes des Gonaïves. C'est là le simulacre fondateur.

Insistons sur le fait que les signatures formelles apposées au bas de l'Acte ne relèvent pas du performatif à ce niveau, puisque les officiers ont signé à partir de leur statut reconnu de chefs de l'armée indigène non ceux de l'armée d'Haïti. «La signature crée les signataires » dit Derrida (1984: 23) au sujet des Représentants américains. Tel n'est pas le cas pour les officiers de l'acte d'indépendance ${ }^{15}$. C'est le peuple qui se crée en signant. Mais, ce faisant, il efface la signature des officiers, car il transforme l'armée indigène en armée d'Haïti, autant dire que l'acte en se constituant détruit et crée, en même temps, les instances de sa production. C'est, à mon avis, l'un de ses points cruciaux de différentiation d'avec la Déclaration d'indépendance des États-Unis. C'est que les Américains ont pris possession de leur terre en position anticipée de parlementaires, alors que les Haïtiens l'ont habitée en militaires d'une armée victorieuse.

Le peuple signe par sa simple présence sur la place d'armes. La présence peut s'étendre à un geste : l'applaudissement. Cela suffit pour qu'il signe. On est sûr que dans sa grande majorité il ne comprend pas

15. La caducité de la signature des généraux est déjà inscrite dans la ligne qui la précède : «Fait aux Gonaïves, ce $1^{\text {er }}$ janvier 1804 et le $1^{\text {er }}$ de l'Indépendance d'Haïti ». L'indépendance détruit l'armée indigène. 
un mot du texte. Mystification ? Là encore, il faut éviter de s'égarer. Nous constituer peuple, c'est nous approprier les modèles de ceux qui nous ont toujours refusé un tel statut, comme le statut d'hommes qui lui est corrélatif. L'écrit, acte fondateur, n'a de registre jusque-là que la langue française. On peut imaginer, faute de détails précis sur la journée du $1^{\text {er }}$ janvier, des voix qui annoncent que le moment décisif va arriver avec la lecture de l'Acte d'indépendance et ce peuple qui, sachant que son adhésion est nécessaire, modèle son comportement sur une attitude d'approbation.

Beaubrun Ardouin, qui considère Boisrond-Tonnerre comme l'un des responsables de la dérive despotique du premier chef d'État haïtien, soutient que si les généraux ne se sont pas opposés à son choix comme rédacteur de l'Acte, c'est pour raison de sagesse : ils évitaient d'entrer prématurément en conflit avec Dessalines. En effet, ce décalage est confirmé par le futur immédiat de la nation haïtienne; l'assassinat du Pont-Rouge est l'aboutissement d'un complot ourdi par presque tous les généraux signataires de l'Acte. Mais il faut pourtant se garder des conclusions hâtives. L'assertion d'Ardouin l'engage avant tout comme citoyen, sujet de l'histoire en cours, non comme historien. C'est le refus de s'inscrire dans l'identité telle qu'elle est définie sous l'empereur et dont il voit le point de départ dans L'Acte ; autant dire que ce qui est remis en cause ici c'est la mêmeté, non l'ipséité (Ricœur 1990). Car on ne saurait nier que le document institue un simulacre de légitimation de soi qui fait intervenir toutes les instances nécessaires à un parcours identificatoire. Ne théâtralise-t-il pas le même et l'autre dans une relation de circularité ?

Ne réduisons pas non plus les préoccupations de Boisrond-Tonnerre à celles de Jefferson. Pour ce dernier, le texte à rédiger devait fournir tous les arguments nécessaires à la justification d'un État américain différent de la Grande-Bretagne ; alors que le rédacteur haïtien circonscrivait l'urgence dans l'établissement de Soi sur la terre, la réalisation d'un désir non encore catalogué comme naturel, celui de signer son humanité. 


\section{Références}

Ardouin, Beaubrun, 1958, Études sur l'histoire d'Haïti. Port-au-Prince :

Docteur François Dalencourt.

Charaudeau, Patrick et Dominique Mainguenau, 2002, Dictionnaire

d'analyse $d u$ discours. Paris, Seuil.

Coquet, Jean-Claude, 1997, La quête du sens. Paris : PUF.

—, 1984, Le Discours et son sujet, tome I. Paris : Klincksieck.

—, 1985, Le Discours et son sujet, tome II. Paris : Klincksieck.

Derrida, Jacques, 1984, Otobiographies. Paris : Galilée.

Jakobson, Roman, 1963, Essais de linguistique générale. Paris : Minuit.

Madiou, Thomas, 1983, Histoire d'Haïti, tome III. Port-au-Prince : Henri Deschamps.

Ricœur, Paul, 1990, Soi-même comme un autre. Paris : Seuil. 